\title{
Spatiotemporal dynamics of emerging pathogens in questing Ixodes ricinus
}

\author{
Elena Claudia Coipan ${ }^{1,2 *}$, Setareh Jahfari ${ }^{1}$, Manoj Fonville ${ }^{1}$, Catharina B. Maassen ${ }^{1,3}$, \\ Joke van der Giessen ${ }^{1,3}$, Willem Takken ${ }^{2}$, Katsuhisa Takumi ${ }^{1}$ and Hein Sprong ${ }^{1,2 *}$ \\ ${ }^{1}$ Centre for Infectious Disease Control, National Institute for Public Health and Environment (RIVM), Bilthoven, Netherlands \\ 2 Laboratory of Entomology, Wageningen University, Wageningen, Netherlands \\ ${ }^{3}$ Food Chain Quality, Antibiotics and Zoonoses Research Group, Central Veterinary Institute, Lelystad, Netherlands
}

Edited by:

Agustín Estrada-Peña, University of

Zaragoza, Spain

Reviewed by:

Max Maurin, Université

Aix-Marseille II, France

Alessandro Mannelli, Dipartimento

di Scienze Veterinarie, Italy

*Correspondence:

Elena Claudia Coipan and Hein

Sprong, National Institute for Public Health and Environment (RIVM),

Centre for Infectious Disease

Control, PO Box 1, 3720 BA,

Bilthoven, Utrecht, Netherlands

e-mail: claudia.coipan@rivm.nl;

hein.sprong@rivm.nl
Ixodes ricinus transmits Borrelia burgdorferi sensu lato, the etiological agent of Lyme disease. Previous studies have also detected Rickettsia helvetica, Anaplasma phagocytophilum, Neoehrlichia mikurensis, and several Babesia species in questing ticks in The Netherlands. In this study, we assessed the acarological risk of exposure to several tick-borne pathogens (TBPs), in The Netherlands. Questing ticks were collected monthly between 2006 and 2010 at 21 sites and between 2000 and 2009 at one other site. Nymphs and adults were analysed individually for the presence of TBPs using an array-approach. Collated data of this and previous studies were used to generate, for each pathogen, a presence/absence map and to further analyse their spatiotemporal variation. $R$. helvetica $(31.1 \%)$ and $B$. burgdorferi sensu lato $(11.8 \%)$ had the highest overall prevalence and were detected in all areas. N. mikurensis (5.6\%), A. phagocytophilum (0.8\%), and Babesia spp. $(1.7 \%)$ were detected in most, but not all areas. The prevalences of pathogens varied among the study areas from 0 to $64 \%$, while the density of questing ticks varied from 1 to $179 / 100 \mathrm{~m}^{2}$. Overall, $37 \%$ of the ticks were infected with at least one pathogen and $6.3 \%$ with more than one pathogen. One-third of the Borrelia-positive ticks were infected with at least one other pathogen. Coinfection of $B$. afzelii with $N$. mikurensis and with Babesia spp. occurred significantly more often than single infections, indicating the existence of mutual reservoir hosts. Alternatively, coinfection of $R$. he/vetica with either B. afzelii or N. mikurensis occurred significantly less frequent. The diversity of TBPs detected in I. ricinus in this study and the frequency of their coinfections with $B$. burgdorferi s.l., underline the need to consider them when evaluating the risks of infection and subsequently the risk of disease following a tick bite.

Keywords: vector-borne disease, Borrelia burgdorferi, Candidatus Neoehrlichia mikurensis, Rickettsia helvetica, Rickettsia conorii, Anaplasma phagocytophilum, Babesia, Ixodes ricinus

\section{INTRODUCTION}

In The Netherlands, the hard tick Ixodes ricinus is the main vector of a variety of human pathogens. The most prevalent tick-borne disease is Lyme borreliosis (Stanek et al., 2012). This multisystemic disorder is caused by several members of the Borrelia burgdorferi sensu lato complex. Of the 18 genospecies of this complex (Margos et al., 2011), B. afzelii, B. garinii, B. spielmanii, $B$. bavariensis, and $B$. burgdorferi sensu stricto have already been detected in The Netherlands, in both patients and questing ticks. B. lusitaniae, and B. valaisiana were detected in questing I. ricinus, but their public health significance is less clear (Collares-Pereira et al., 2004; Diza et al., 2004; De Carvalho et al., 2008; Coipan et al., 2013).

Over the last decades, the incidence of Lyme borreliosis has increased significantly in Europe (Smith and Takkinen, 2006). A long-term retrospective study among general practitioners in The Netherlands has shown a continuing increase in consultations for tick bites and erythema migrans in the last decade (Hofhuis et al., 2006). The incidence of erythema migrans patients increased from
39 per 100,000 inhabitants in 1994 to 134 per 100,000 inhabitants in 2009.

Previous studies in The Netherlands have identified the presence of other pathogens in questing I. ricinus as well. Human babesiosis is caused by the intraerythrocytic protozoa Babesia divergens, B. venatorum (EU1), and B. microti (Vannier and Krause, 2012). A recent study reported these three Babesia species in approximately $1 \%$ of questing $I$. ricinus (Wielinga et al., 2009). The spotted fever syndrome is caused by at least 15 different Rickettsia species, some of which are transmitted by I. ricinus (Heyman et al., 2010). Rickettsia conorii and R. monacensis are probably the most common tick-borne Rickettsiae to cause disease in Europe (Heyman et al., 2010), whereas the pathogenicity of $R$. helvetica is still questionable (Svendsen, 2011). All three rickettsial species have been previously found in The Netherlands (Sprong et al., 2009) with local prevalences varying from $<1 \%$ ( $R$. conorii) to as high as $66 \%$ ( $R$. helvetica). Anaplasma phagocytophilum, the etiologic agent of human granulocytotropic anaplasmosis (Sprong et al., 2009), has been detected 
in Dutch ticks in several studies (Tijsse-Klasen et al., 2010, 2011b). Neoehrlichia mikurensis can be considered an emerging zoonosis, as more than eight human cases have been described in Europe since 2010, while previously it was considered non-pathogenic. Despite an overall prevalence of $N$. mikurensis in questing ticks of approximately $7 \%$ (Jahfari et al., 2012), no human cases have been reported in The Netherlands.

Autochthonous tick-borne diseases other than Lyme disease have not been reported, except for a single case of granulocytotropic anaplasmosis in 1999 (Van Dobbenburgh et al., 1999). This may be caused by lower pathogenicity, lack of overt symptoms, or lack of awareness of public and health professionals.

Multiple studies have reported coinfection with some of the tick-borne pathogens (TBPs) (Belongia, 2002; Ginsberg, 2008; Nieto and Foley, 2009; Reye et al., 2010; Burri et al., 2011; Lommano et al., 2012). It is known that the severity of Lyme disease may be affected by simultaneous infections with other TBPs (Belongia, 2002; Swanson et al., 2006). Some of them, such as $A$. phagocytophilum, modulate host immunity and increase susceptibility to various second pathogens, including B. burgdorferi (Thomas et al., 2001; Holden et al., 2005). Thus, coinfection might be partly responsible for the variability in clinical manifestations that are usually associated with Lyme borreliosis.

The acquirement of a tick-borne disease depends on many environmental, societal, and immunological factors, but it is always preceded by the bite of a tick infected with the causal agent. Previous studies have shown that the risk of infection of humans by TBPs depends mainly on the density of questing infected ticks—-the acarological risk (Glass et al., 1994, 1995; Nicholson and Mather, 1996; Dister et al., 1997; Kitron and Kazmierczak, 1997). The study of mixed infections in questing ticks might, therefore, reveal patterns of coinfection of B. burgdorferi s.l. with two or more other pathogens, allowing us to generate hypotheses on the transmission cycle of some more obscure pathogens from the dynamics of better-known ones. The aim of this study was to assess the acarological risk of exposure to TBPs in The Netherlands by comparing the abundances of questing ticks infected with B. burgdorferi s.l. and with other TBPs.

\section{METHODS}

\section{COLLECTION OF TICKS AND TICK DATA}

All ticks were collected on a monthly basis between 2006 and 2010 in 21 sites. In Duin\&Kruidberg field sampling was conducted between 2000 and 2009. The sites were spread all over The Netherlands and they have been selected based on Lyme borreliosis incidence in humans, and the availability of volunteers. The same sites were described in some previous studies regarding ticks and TBPs in The Netherlands (Wielinga et al., 2006, 2009; Sprong et al., 2009; Gassner et al., 2011; Tijsse-Klasen et al., 2011a). Sampling of ticks was done by blanket dragging, using a $1 \mathrm{~m}^{2}$ cloth on a $100 \mathrm{~m}$ long transect. Based on morphological criteria, ticks were identified to species level, with stage and sex recorded. The density of ticks was estimated as the number of questing ticks per $100 \mathrm{~m}^{2}$. Additionally, data on the presence of ticks and TBPs in other 39 areas were collected from some previous studies that have used the same sampling and analysis methodology (Schouls et al., 1999; Tijsse-Klasen et al., 2010, 2011b; Jahfari et al., 2012).

\section{DNA EXTRACTION OF TICKS}

All the collected ticks were immersed in $70 \%$ alcohol and stored at $-20^{\circ} \mathrm{C}$ until the DNA extraction. DNA from questing ticks was extracted by alkaline lysis (Wielinga et al., 2006). Questing larvae were not taken into account as humans are generally bitten by either nymphs or adult I. ricinus (Hugli et al., 2009; Tijsse-Klasen et al., 2011b).

\section{PCR DETECTION AND IDENTIFICATION OF PATHOGENS}

The presence of the DNA of different TBPs (Rickettsia spp., B. burgdorferi s.l., Ehrlichia/Anaplasma spp., and Babesia spp.) was determined by polymerase chain reaction (PCR) followed by reverse line blotting (RLB) as described before (Wielinga et al., 2006; Tijsse-Klasen et al., 2010). To minimize cross contamination and false-positive results, positive and negative controls were included in each batch tested by PCR and RLB assays. Furthermore, DNA extraction, PCR mix preparation, sample addition, and PCR analysis were performed in assigned separate labs. PCR products of some samples were sequenced by dideoxy-dye termination sequencing of both strands, and compared with sequences in GenBank (http://www.ncbi.nlm.nih. gov/), using BLAST (Altschul et al., 1990). The sequences were aligned and analysed using BioNumerics 6.6 (Applied Maths, Kortrijk, Belgium).

The prevalence of infection was calculated as the percentage of ticks infected with a certain microorganism.

\section{ACAROLOGICAL RISK}

To calculate the densities of questing ticks infected with each of the five pathogens' genera, we multiplied the prevalence of infection with the density of questing ticks in each of the investigated sites.

\section{CORRELATION BETWEEN PREVALENCE AND TICK DENSITY}

For some pathogens, we noticed that the prevalence might correlate with the density of questing ticks at the sampling locations. To test this possibility we fitted a binomial model to our data, by defining the prevalence of infection as an exponential function of the tick density $(d)$ at each sampling location. Knowing that the number of infected ticks $(k)$ out of the total number of ticks tested $(n)$, is binomially distributed with a probability $(p)$, we used the function $p=a \operatorname{Exp}[b d], 0<a<1$, to test an alternative model $(b<0)$ against a null model $(b=0)$ by a likelihood-ratio test. The alternative (decreasing exponential) model fitted significantly better to our data with $p$-value $P \leq 0.05$.

\section{SEASONAL DYNAMICS}

To test for the seasonal dynamics of infection in ticks, a binomial model for the probability of infection $(p)$ was fitted to our data, in combination with the sampling days $(t)$ and pooled over the sampling locations and development stages. The probability of infection was a logit function $p=\frac{e^{y}}{1+e^{y}}$ and we modeled seasonality by using the trigonometric function $y=a+b \cos (2 \pi t / 365)+$ 
$c \sin (2 \pi t / 365)$ to describe an oscillation with a period of 1 year and possible phase shift. For each pathogen, we tested the seasonal model against the non-seasonal model $(y=a)$ based on a likelihood ratio test. The seasonal model fitted significantly better to our data with $p$-value $P \leq 0.05$. All the statistical analyses were performed using Wolfram Mathematica 9.

\section{RESULTS}

The mean density of questing nymphs and adult ticks varied greatly between sites, from as low as 1 (at Houtvesterijen Heide) up to $179 / 100 \mathrm{~m}^{2}$ (at Duin\&Kruidberg; Table 2), results that are consistent with previous Dutch studies (Wielinga et al., 2006).

\section{PATHOGEN DETECTION AND IDENTIFICATION}

A total of 5570 questing nymphs and adult $I$. ricinus from 22 different study areas were tested for the presence of TBPs by PCR amplification followed by RLB (Table 1). The recently identified $B$. bavariensis reacted consistently with our B. garinii probe (Margos et al., 2009), and therefore we grouped these two Borrelia genospecies. Five Borrelia genospecies were found in this study in all twenty-two study areas (Table 1), with the overall prevalence $(11.8 \%)$ inscribed in the interval of average European prevalence (Rauter and Hartung, 2005), and comparable with previous studies in The Netherlands (Wielinga et al., 2006; Gassner et al., 2011). B.afzelii was predominant (6.7\%), followed by B. garinii/B. bavariensis (1.5\%), B. valaisiana (1.2\%), and $B$. burgdorferi sensu stricto $(0.2 \%)$. The remaining fraction of the Borrelia positive samples could not be further identified to the species level by RLB. Sequencing several of these samples revealed the presence of B. spielmanii, corroborating previous findings of this genospecies in The Netherlands (Wang et al., 1999). B. lusitaniae, which was recently found in The Netherlands (Tijsse-Klasen et al., 2010), was not detected in this study.

$R$. helvetica was most frequently detected in tick lysates, its $31.1 \%$ average prevalence (Table 1) being among one of the highest in Europe [range 1.5 to more than $40.6 \%$ (Christova et al., 2003; Severinsson et al., 2010)]. A previous study from our laboratory found $R$. helvetica not only in vertebrate hosts, but also in tick larvae at comparable prevalences as for the other tick stages, indicating a high efficiency of transovarial transmission (Sprong et al., 2009). Thirty-three Rickettsia isolates could not be identified up to the species level by RLB. Sequencing of these samples revealed the presence of $R$. monacensis, which was reported in The Netherlands before (Sprong et al., 2009). Rickettsia conorii was detected in only three questing ticks from one study area (Veldhoven). A. phagocytophilum-infected ticks were recorded with an overall prevalence of only $0.8 \%$ (Table 1 ). N. mikurensis DNA was found with a global prevalence of 5.6\% (Table 1). Ehrlichia canis DNA was detected in only 5 tick lysates from four different study areas, which resulted in an overall prevalence of $0.1 \%$ (5/5343). Ninety-nine Ehrlichia isolates could not be identified to the species level neither by RLB nor by sequencing. Babesia venatorum, formerly also known as B.EU1 (Duh et al., 2005), was present with a global prevalence of $1.0 \%(41 / 4238)$. The prevalence of $B$. microti in questing ticks was $0.4 \%$ (17/4238), and the

Table 1 | Presence of microorganisms in questing $I$. ricinus nymphs and adults.

\begin{tabular}{|c|c|c|c|c|c|}
\hline Pathogen & Positive/tested $I$. ricinus (n) & Prevalence I. ricinus (\%) & \multicolumn{2}{|c|}{$95 \% \mathrm{CL}$} & Tested/\%positive study areas \\
\hline Borrelia burgdorferi s.I. & $628(5308)$ & 11.8 & 11.0 & 12.7 & $22 / 100$ \\
\hline -Borrelia afzelii (and B.ruski) & 355 (5308) & 6.7 & 6.0 & 7.4 & $22 / 100$ \\
\hline -Borrelia garinii/B.bavariensis & 79 (5308) & 1.5 & 1.2 & 1.9 & $22 / 77.3$ \\
\hline -Borrelia valaisiana & $64(5308)$ & 1.2 & 0.9 & 1.5 & $22 / 81.8$ \\
\hline Rickettsia helvetica & $1265(4061)$ & 31.1 & 29.7 & 32.6 & $19 / 100$ \\
\hline Rickettsia conorii & $3(4061)$ & 0.1 & 0.0 & 0.2 & $19 / 5.3$ \\
\hline Untypeable Rickettsia & $33(4061)$ & 0.8 & 0.6 & 1.1 & 19/68.4 \\
\hline Anaplasma phagocytophilum & $44(5343)$ & 0.8 & 0.6 & 1.1 & 22/63.6 \\
\hline Neoehrlichia mikurensis & 300 (5343) & 5.6 & 5.0 & 6.3 & $22 / 81.8$ \\
\hline Babesia venatorum (EU1) & $41(4238)$ & 1.0 & 0.7 & 1.3 & $19 / 73.7$ \\
\hline Babesia divergens & $1(4238)$ & 0.0 & 0.0 & 0.1 & $19 / 5.3$ \\
\hline Untypeable Babesia & $12(4238)$ & 0.3 & 0.2 & 0.5 & 19/63.2 \\
\hline
\end{tabular}

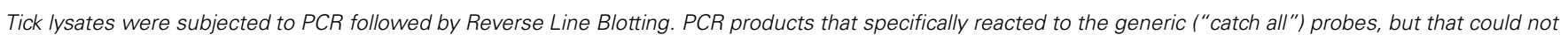

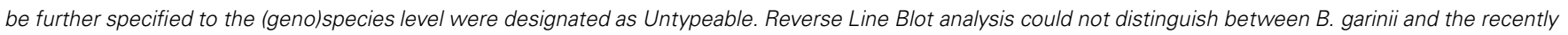
identified B. bavariensis. Calculations of prevalence were based on all tick lysates that were analysed (n).

$C L$, confidence limits; $L C L$, lower confidence limit; UCL, upper confidence limit. 

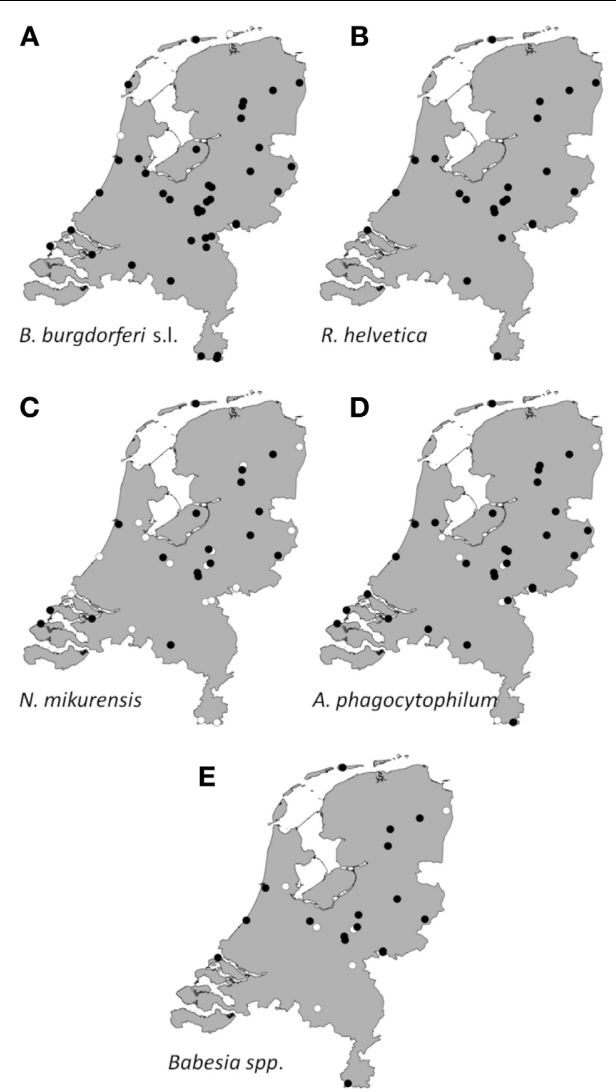

FIGURE 1 | Aggregated presence/absence map of questing $l$. ricinus nymphs/adults infected with $B$. burgdorferi s.I. (A), R. helvetica (B), N. mikurensis (C), A. phagocytophilum (D), Babesia species (E). The black dots represent presence of the microorganism; the white ones represent absence. Presence/absence points from previous studies were also incorporated.

protozoan was detected in 6 from 19 sites. Only one tick from the Duin\&Kruidberg area contained the DNA of previously reported B. divergens (Wielinga et al., 2009). Twelve Babesia sp. could not be further identified by neither RLB, or sequencing. The average prevalence of Babesia-positive ticks in the study areas was $1.6 \%$ (Table 1).

\section{SPATIAL DISTRIBUTION AND VARIATION}

Collated data were used to generate presence/absence maps of the five major TBPs in The Netherlands (Figure 1). The presence/absence of Borrelia spp., $R$. helvetica, A. phagocytophilum, N. mikurensis, and Babesia spp. was assessed for 61, 24, 39, 39, and 25 locations, respectively. The presence of these pathogens was observed in 58, 24, 33, 20 and 18 areas, respectively, heterogeneously distributed across The Netherlands. The few absence points were scattered over The Netherlands as well, and did not cluster in any geographic region (Figure 1).

Borrelia prevalence was between 5\% (Houtvesterijen Heide) and 50\% (Bellingwedde; where only six ticks were tested), while for $R$. helvetica it varied even more, from 3\% in some sites (Apeldoorn), to $64 \%$ in others (Duin\&Kruidberg) (Table 2).
Lower variations in prevalences were observed for $N$. mikurensis, A. phagocytophilum and Babesia spp. (Table 2). For N. mikurensis, the prevalence was on average of $5 \%$, but some areas displayed values of over $10 \%$ (Table 2). Babesia spp. showed an overall prevalence of $1.7 \%$, similarly to Germany and Luxembourg (Hartelt et al., 2004; Reye et al., 2010). A. phagocytophilum was the least prevalent pathogen in our study, with a mean prevalence of $0.8 \%$ - comparable with the $0.5-1 \%$ prevalence found in different European countries (Koci et al., 2007; Hildebrandt et al., 2010; Severinsson et al., 2010). However, one of the sites displayed a 10 -fold higher prevalence than average (Bilthoven $8 \%$, Table 2).

Identification of high risk-areas depends on both pathogen prevalence and density of questing ticks (nymphs and adults). The density of questing ticks varied between $1 / 100 \mathrm{~m}^{2}$ (Houtvesterijen Heide) and 179/100 $\mathrm{m}^{2}$ (Duin\&Kruidberg; Table 2). The density of questing Borrelia-infected ticks varied between 0 and 19 ticks per $100 \mathrm{~m}^{2}$ (Figure 2), whereas the maximum densities of A. phagocytophilum, N. mikurensis and Babesia spp. infected ticks were $3.0,13$, and 2.9 ticks per $100 \mathrm{~m}^{2}$, respectively. The density of questing $R$. helvetica-infected ticks varied between 0 and 22 ticks per $100 \mathrm{~m}^{2}$, with one notable exception: Duin\&Kruidberg area had both a high tick density and an exceptionally high $R$. helvetica prevalence, which resulted in a density of questing $R$. helveticainfected ticks of up to 119 ticks per $100 \mathrm{~m}^{2}$. Considering that these are calculated as average values for an entire season, it is therefore inevitable that the densities of infected questing ticks are actually higher for peak months of tick activity [i.e., May-June (Gassner et al., 2011)].

Based on a likelihood ratio test, performed for a decreasing model and a constant one, we detected a significant negative correlation between the density of questing ticks and the infection prevalence with B. burgdorferi s.l. $\left(p=3.6 \times 10^{-10}\right)$ and Babesia spp. $\left(p=4.9 \times 10^{-5}\right)$ (Figure 3). On the other hand, there was no correlation found between these variables for $R$. helvetica $(p=1.0), N$. mikurensis $(p=1.0)$ and A. phagocytophilum ( $p=$ $0.69)$ (Figure 3). Graphs for the density of infected questing ticks against the density of questing ticks revealed that the former is linearly increasing with the latter for R. helvetica, N. mikurensis and A. phagocytophilum (Figure 4). For the other two pathogensBabesia spp. and B. burgdorferi s.l., the density of infected questing ticks reached the maximum values at densities of questing ticks of 119 and 268, respectively (Figure 4).

\section{TEMPORAL VARIATION}

To gain insight into long-term dynamics of ticks and their pathogens, we analysed the data obtained from Duin\&Kruidberg, where a 10-year (2000-2009) tick-surveillance was performed. This area was selected at that time because of its unusual high tick density/activity. The prevalences of all pathogens were relatively stable over the past decade (B. burgdorferi s.l. $7.0 \%$, B. afzelii $4.6 \%$, A. phagocytophilum $0.7 \%, R$. helvetica $65 \%$, Babesia spp. $1.1 \%$ ), except for N. mikurensis, whose prevalence increased from $3.5 \%(2000-2007)$ to $12 \%$ in the last 2-year interval (2008-2009). The average density/activity of adult ticks remained relatively low with $7-34$ ticks per $100 \mathrm{~m}^{2}$. The average density/activity of nymphal ticks was more pronounced 
Table 2 | Prevalence (\%) of the five major pathogens found in the 22 study areas.

\begin{tabular}{|c|c|c|c|c|c|c|c|c|c|c|c|c|c|c|c|c|c|}
\hline $\begin{array}{l}\text { Geographic } \\
\text { location }\end{array}$ & \multicolumn{3}{|c|}{ B. burgdorferi s.I. } & \multicolumn{3}{|c|}{ R. helvetica } & \multicolumn{3}{|c|}{ N. mikurensis } & \multicolumn{3}{|c|}{ A. phagocytophylum } & \multicolumn{3}{|c|}{ Babesia spp. } & \multicolumn{2}{|c|}{ Density $\left(/ 100 \mathrm{~m}^{2}\right)$} \\
\hline Apeldoorn & 15 & 38 & 39 & 1 & 38 & 3 & 3 & 38 & 8 & 0 & 38 & 0 & 5 & 38 & 13 & 17 & 5 \\
\hline Appelscha & 10 & 79 & 13 & 11 & 76 & 14 & 3 & 79 & 4 & 0 & 79 & 0 & 4 & 79 & 5 & 19 & 5 \\
\hline Bellingwedde & 3 & 6 & 50 & 2 & 6 & 33 & 0 & 6 & 0 & 0 & 6 & 0 & 0 & 6 & 0 & 7 & 0.3 \\
\hline Bijlmerweide & 34 & 330 & 10 & & & ND & 1 & 330 & 0.3 & 0 & 330 & 0 & & & ND & 12 & 1 \\
\hline Ede & 48 & 354 & 14 & 23 & 354 & 6 & 36 & 353 & 10 & 3 & 353 & 1 & 2 & 353 & 1 & 54 & 7 \\
\hline Eijsden & 28 & 232 & 12 & 23 & 232 & 10 & 0 & 232 & 0 & 1 & 232 & 0.4 & 10 & 232 & 4 & 34 & 1 \\
\hline Gieten & 10 & 136 & 7 & 31 & 136 & 23 & 6 & 136 & 4 & 2 & 136 & 1 & 2 & 136 & 1 & 59 & 5 \\
\hline Haaksbergen & 9 & 105 & 9 & 11 & 105 & 10 & 1 & 105 & 1 & 4 & 105 & 4 & 2 & 105 & 2 & 77 & 2 \\
\hline Houtvest_Heide & 4 & 88 & 5 & & & ND & 5 & 88 & 6 & 1 & 88 & 1 & & & ND & 1 & 0.2 \\
\hline Kwade Hoek & 43 & 162 & 27 & 13 & 162 & 8 & 23 & 162 & 14 & 0 & 162 & 0 & 3 & 162 & 2 & 9 & 4 \\
\hline Montferland & 18 & 1470 & 12 & 12 & 147 & 8 & 11 & 147 & 7 & 0 & 147 & 0 & 3 & 147 & 2 & 40 & 3 \\
\hline Nijverdal & 24 & 127 & 19 & 13 & 127 & 10 & 18 & 127 & 14 & 1 & 127 & 1 & 8 & 127 & 6 & 34 & 2 \\
\hline Ruinen & 25 & 94 & 27 & 30 & 94 & 32 & 2 & 94 & 2 & 2 & 94 & 2 & 1 & 94 & 1 & 18 & 1 \\
\hline Twiske & 46 & 292 & 16 & 62 & 292 & 21 & 13 & 292 & 4 & 1 & 292 & 0.3 & 0 & 292 & 0 & 36 & 2 \\
\hline Veldhoven & 25 & 242 & 10 & 14 & 239 & 6 & 13 & 242 & 5 & 6 & 242 & 2 & 1 & 242 & 0.4 & 47 & 17 \\
\hline Wassenaar & 33 & 204 & 15 & 91 & 204 & 45 & 4 & 204 & 2 & 1 & 204 & 0 & 3 & 204 & 1 & 46 & 3 \\
\hline Total/Average & 628 & 5308 & 11.8 & 1265 & 4061 & 31.1 & 300 & 5343 & 5.6 & 44 & 5343 & 0.8 & 71 & 4238 & 1.7 & 38 & 4 \\
\hline
\end{tabular}

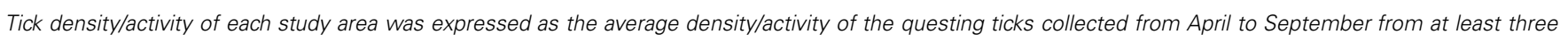
consecutive years. Average prevalences of the study areas $(n=22)$ were calculated. + , positive samples; $T$, tested; ND, Not determined.

(102-410 ticks per $100 \mathrm{~m}^{2}$ ) and peaked in 2004-2005 (Figure 5). The likelihood ratio test detected similar decreasing trends in the temporal relation between the prevalence and the tick density as for the spatial variation analysis (not shown). Despite the inverse relationship between the prevalence and the tick density, the peaks of density/activity of infected ticks coincided with the peak of high densities of questing ticks in 2004-2005 (Figure 5).

\section{COINFECTION}

Overall, 37\% (2064/5570) of the ticks was infected with one or more pathogens and $6.3 \%(350 / 5570)$ with more than one pathogen of different genera. Furthermore, 37\% (234/628) of the Borrelia-positive ticks were infected with at least one other pathogen of a different genus. Almost 5\% (29/628) of the Borrelia-positive ticks were also positive for three or more other pathogens. One tick carried the DNA of B.afzelii, R. helvetica, N. mikurensis, and B.microti. Mixed infections, involving two or three Borrelia genospecies, occurred in only $0.3 \%$ (15/5308) of the tick lysates. Coinfection of B. afzelii with $N$. mikurensis or with Babesia spp. occurred significantly more than random, whereas infection of $R$. helvetica with either B.afzelii or $N$. mikurensis occurred significantly less frequent (Table 3).

\section{SEASONAL DYNAMICS}

Seasonality modeling of the prevalence indicated a different periodicity of the analysed pathogens (Figure 6). Thus, B. afzelii, N. mikurensis and Babesia spp. showed highest prevalences in ticks at time periods corresponding to October, while nonafzelii $B$. burgdorferi and $R$. helvetica had the highest prevalence around June. Annual prevalence of A. phagocytophilum was not seasonal.

\section{DISCUSSION}

In order to assess the acarological risk of acquiring a tick-borne infection in The Netherlands, the abundance of questing ticks infected with $B$. burgdorferi s.l. and four other genera of TBPs were compared.

Our study revealed the nationwide circulation of TBPs in enzootic cycles. Although the most common tick-borne infection is acknowledged to be Lyme borreliosis, our results showed that there are other pathogens present in questing ticks at prevalences comparable with B. burgdorferi (i.e., R. helvetica, Table 2). Due to the fact that our investigations only detected the DNA of the microorganisms under discussion, and not the viable cells, we cannot asseverate their infectiousness for other vertebrate hosts. However, previous studies implicate Ixodes ricinus ticks as vectors for these microorganisms (Barbour et al., 1983; Ackermann et al., 


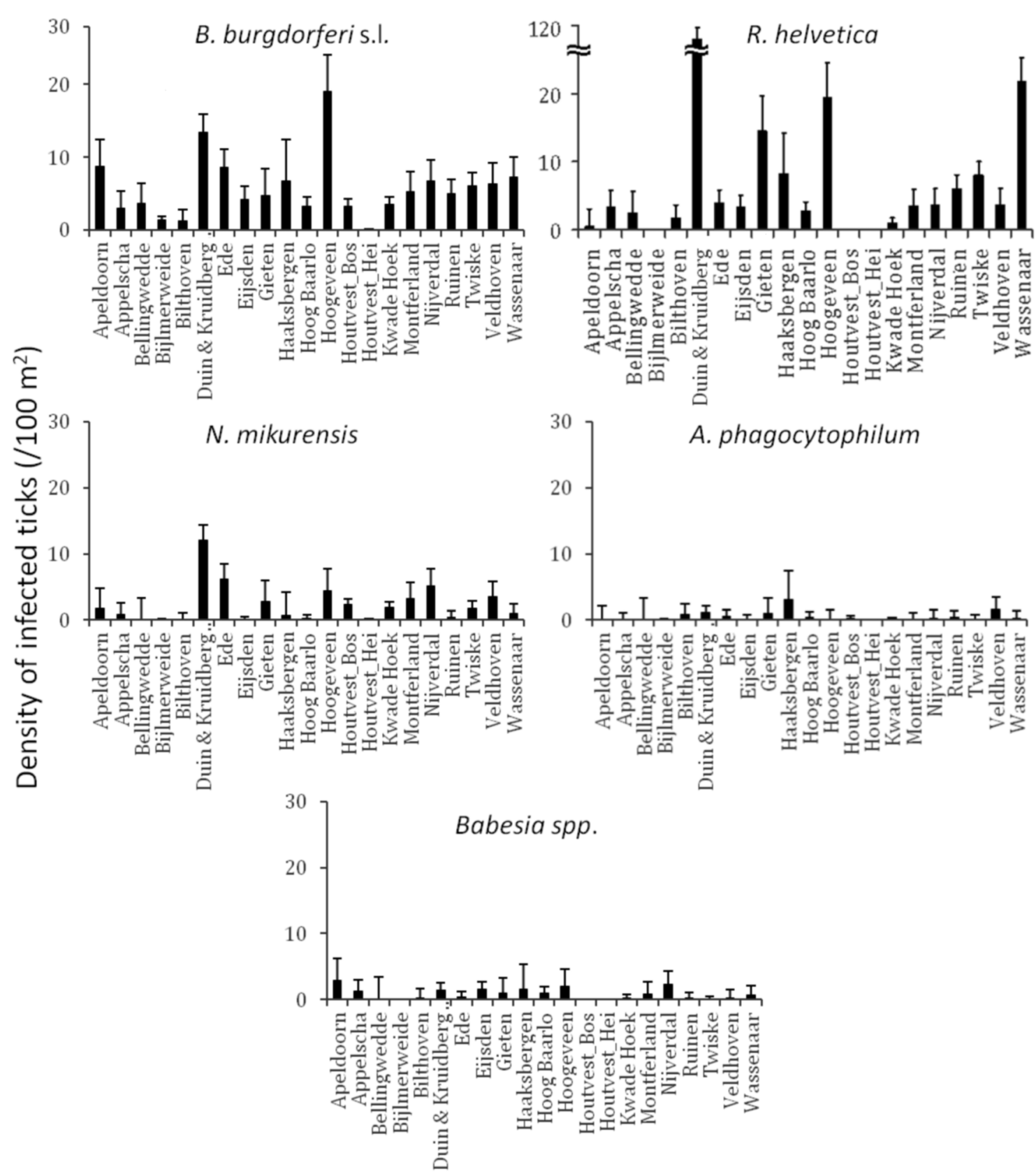

FIGURE 2 | Identification of high risk-areas depends on both prevalence and tick density/activity. Their calculated product defines the density/activity of infected ticks (nymphs and adults/100 $\mathrm{m}^{2}$ ). The error bars depict the upper limit of the $95 \%$ confidence interval. Duin\&Kruidberg's density of $R$. helvetica infected ticks reaches to $119 / 100 \mathrm{~m}^{2}$
1984; Lotric-Furlan et al., 1998; Rydkina et al., 1999; Kjemtrup and Conrad, 2000; Parola and Raoult, 2001; Gray et al., 2002; Bonnet et al., 2007; Sprong et al., 2009; Heyman et al., 2010; Jahfari et al., 2012), and therefore the risk for public health should not be neglected. Although no human disease with the organisms other than B. burgdorferi s.l. was reported so far in The Netherlands, it is known that infection with some of th0em (e.g., Ehrlichia) is generally either asymptomatic or mild, self-limiting diseases (Ismail et al., 2010).

\section{SPATIAL DISTRIBUTION AND VARIATION}

All the pathogens were observed in most of the areas in which investigations were conducted, regardless of the geographical position. The absence in certain areas might be explained by the relatively low number of ticks collected/tested (Table 2). The prevalences of infection in the ticks varied significantly between the areas investigated. The lack of a full perspective on the host community at each of the sites does not allow us to make a definite statement on why we see such a variation of the prevalence 


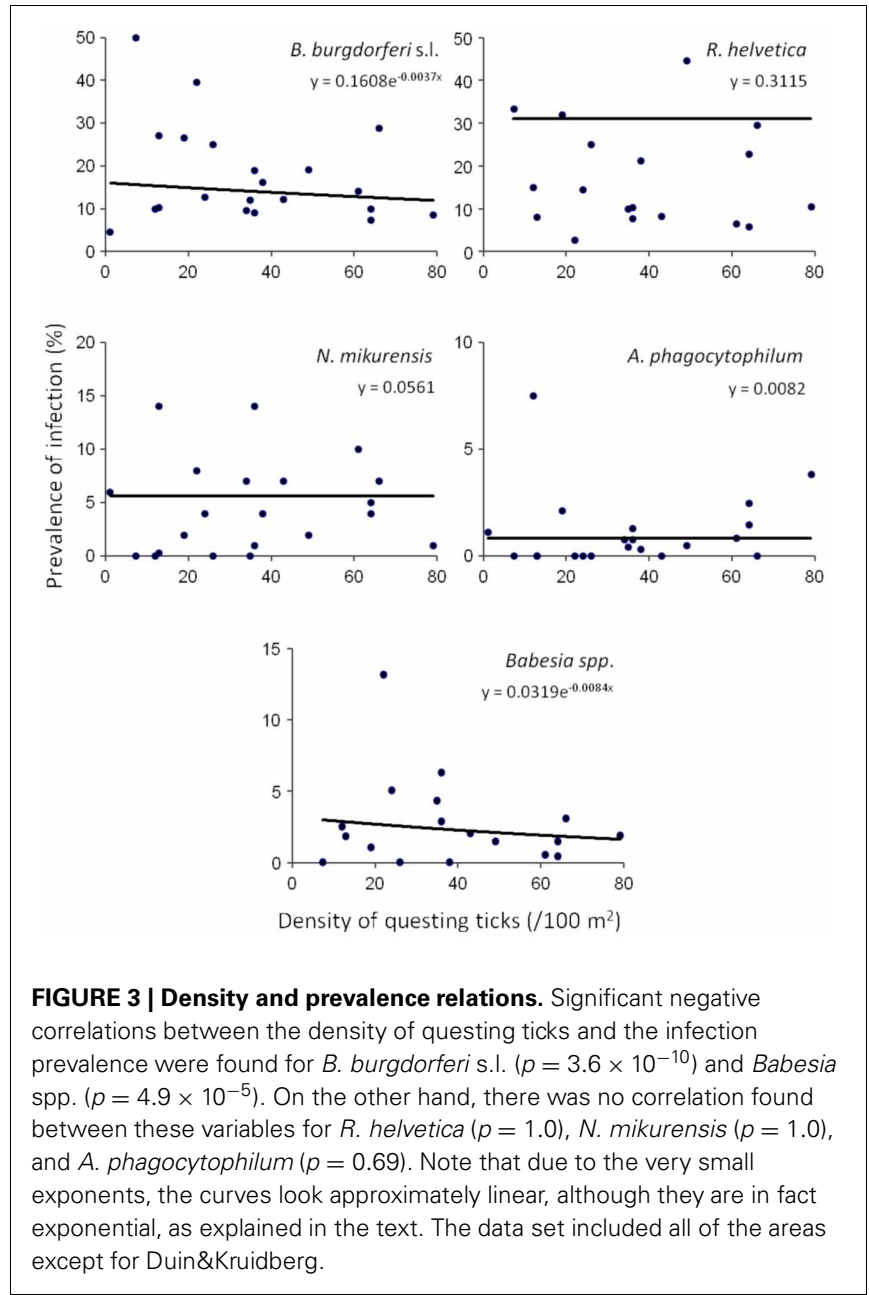

of infection. We propose, however, that the extremely high local variability of the pathogens may be associated with the differences in host assemblages in the investigated habitats. As ticks can feed on many different animals and every host species has a unique reservoir competence [e.g., rodents being the most competent reservoirs of B. afzelii (Gern and Humair, 2002)], the presence of different hosts in different communities affects the prevalence of infection with various microorganisms.

In terms of the risk for public health, neither the density of questing ticks, nor the prevalence of infection alone, has any significance. Instead, it is their product-the density of infected questing ticks - that defines high or low risk areas (Glass et al., 1994, 1995; Nicholson and Mather, 1996; Dister et al., 1997; Kitron and Kazmierczak, 1997). We noticed that in some areas, where tick densities were highest, the mean prevalence of Borrelia infection had very low values ( $8 \%$ for Duin\&Kruidberg; Table 2). Using a log-likelihood ratio statistics, we tested the hypothesis of a constant prevalence over the range of questing ticks density. The test confirmed the independence of the two variables but only for $R$. helvetica, N. mikurensis, and A. phagocytophilum, while for B. burgdorferi and Babesia spp. it indicated a slight negative correlation of the prevalence with the tick density (Figure 3). Thus, we would expect that the density of ticks
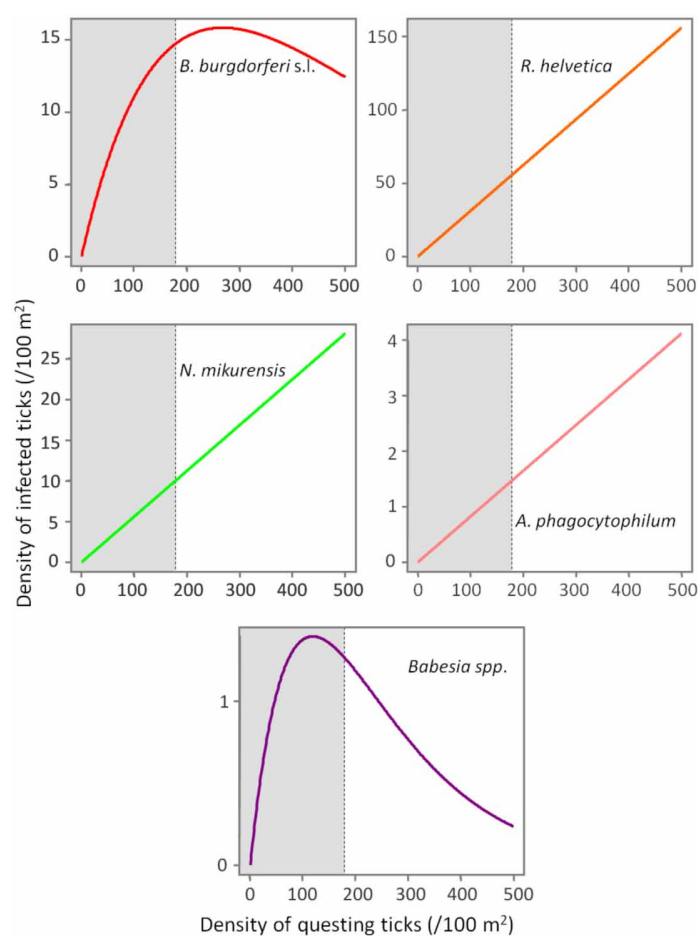

FIGURE 4 | Evolution of the density of infected ticks (y-axis) with the density of questing ticks (x-axis). The density of infected ticks is obtained by fitting a model ( $p=a E x p[b d])$ to a range of questing ticks densities. The numbers are expressed as ticks $/ 100 \mathrm{~m}^{2}$. The gray area marks the normal questing ticks densities $\left(0-179 / 100 \mathrm{~m}^{2}\right)$ in The Netherlands.

infected with B. burgdorferi and Babesia spp. would decrease as the density of questing ticks increases. Plotting the density of infected questing ticks as an exponential function of the questing ticks' densities, however, revealed that over the usual range of questing ticks densities the density of infected ticks is also increasing, and the downward trend might be observed only for questing ticks densities of over 100 (for Babesia spp.) or $200 / 100 \mathrm{~m}^{2}$ (for B. burgdorferi) (Figure 4). This observation is consistent with the finding made by Randolph (Randolph, 2001) that, in Europe the density of Borrelia infected ticks depends much more on the density of all ticks than on the infection prevalence, and that only in areas where the tick density is unusually high $\left(100-450 / 100 \mathrm{~m}^{2}\right)$ is the infection prevalence consistently low.

\section{TEMPORAL VARIATION}

In terms of temporal variation, the longest series of data we had was for 10 successive years (Duin\&Kruidberg, Figure 5). At this site, the density of questing ticks was highest in 2004-2005, and it was due to a steep increase in the number of questing nymphs. The variations in tick density might indicate yearly fluctuations in the composition and availability of reservoir hosts. For example, a mast year might have been responsible for the increment in small mammals' population size (i.e., rodents), with the upsurge of nymphs at a consequential rate. The trend line indicated the maintenance of relatively constant 


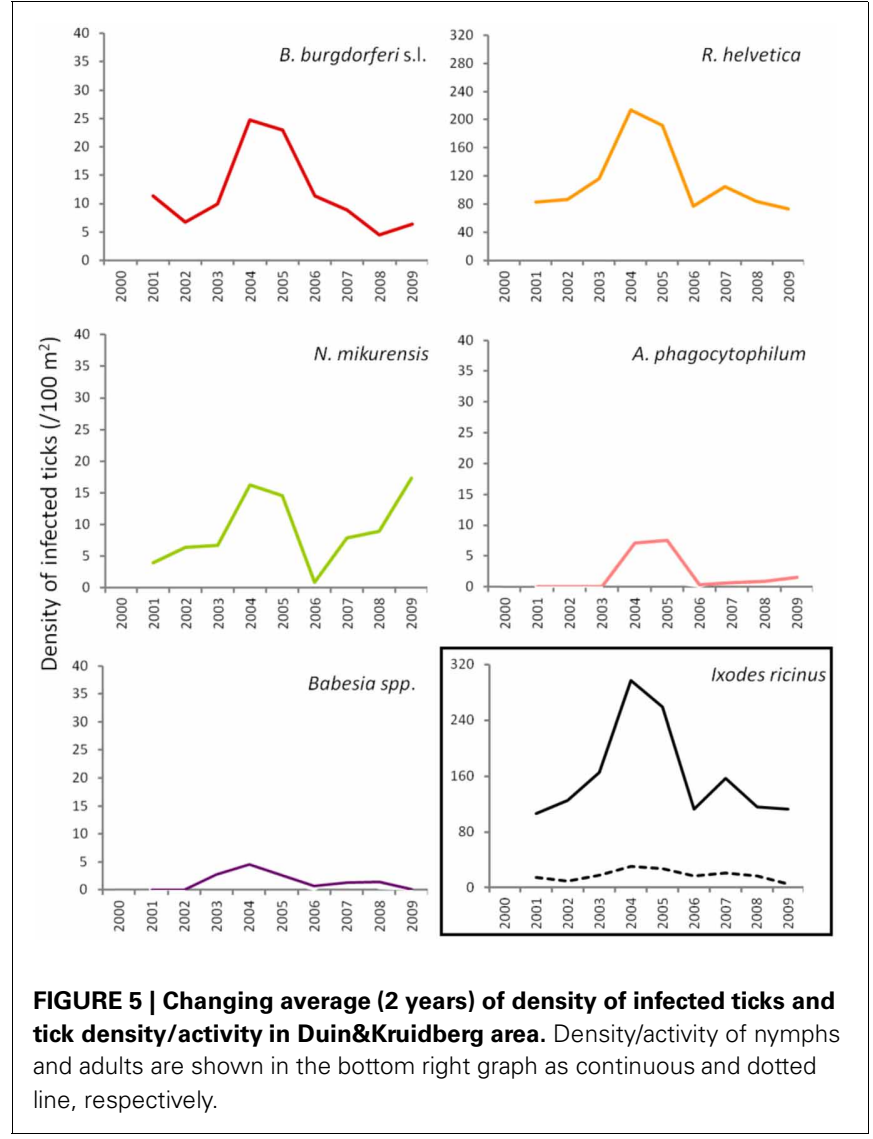

Table 3 | Observed and expected coinfections.

\begin{tabular}{|c|c|c|c|c|}
\hline & R. helvetica & $\begin{array}{l}\text { A. phago- } \\
\text { cytophilum }\end{array}$ & $\begin{array}{l}\text { N. miku- } \\
\text { rensis }\end{array}$ & $\begin{array}{l}\text { Babesia } \\
\text { spp. }\end{array}$ \\
\hline \multicolumn{5}{|l|}{ OBSERVED (\%) } \\
\hline Borrelia (all) & 3.3 & 0.1 & 1.6 & 0.4 \\
\hline B. afzelii & 1.8 & 0.0 & 1.3 & 0.3 \\
\hline R. helvetica & & 0.3 & 2.2 & 0.5 \\
\hline A. phagocytophilum & & & 0.0 & 0.0 \\
\hline N. mikurensis & & & & 0.1 \\
\hline \multicolumn{5}{|l|}{ EXPECTED (\%) } \\
\hline Borrelia (all) & 3.9 & 0.1 & 0.7 & 0.2 \\
\hline B. afzelii & 2.2 & 0.1 & 0.4 & 0.1 \\
\hline R. helvetica & & 0.3 & 1.9 & 0.5 \\
\hline A. phagocytophilum & & & 0.0 & 0.0 \\
\hline N. mikurensis & & & & 0.1 \\
\hline \multicolumn{5}{|l|}{$\chi^{2}$-TEST ( $p$-VALUE) } \\
\hline Borrelia (all) & $0.03^{* *}$ & 0.30 & $0.00^{*}$ & $0.01 *$ \\
\hline B. afzelii & $0.03^{* *}$ & 0.24 & $0.00 *$ & $0.00 *$ \\
\hline R. helvetica & & 0.80 & $0.05^{* *}$ & 0.77 \\
\hline A. phagocytophilum & & & 0.10 & 0.42 \\
\hline N. mikurensis & & & & 0.66 \\
\hline
\end{tabular}

$\chi^{2}$-tests were used to calculate the associations of several combinations of pathogens. ${ }^{*}$ Significant positive associations and ${ }^{* *}$ significant negative associations $(p<0.05)$ are shown in bold.

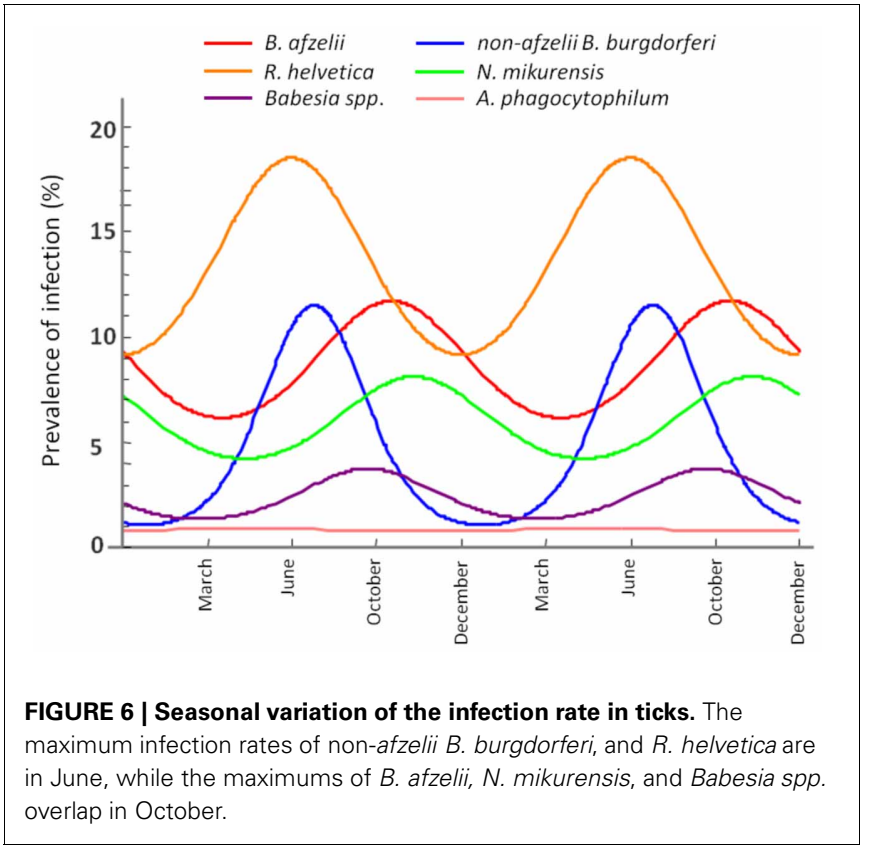

prevalences for B. burgdorferi, A. phagocytophilum, and $R$. helvetica.Babesia prevalence showed a slight decrease over time while, on the contrary, N. mikurensis showed a steep increase (almost 3-fold). The maintenance of relatively constant prevalences of infection in time implies that the acarological risk is predominantly dependent on the density/activity of ticks (Figure 5).

\section{COINFECTION}

One-third of the ticks infected with Borrelia were also infected with at least one other TBP. Recent studies in other European countries have shown that mixed infections of the TBPs do not represent an exception but more likely the rule.

A negative significant association was found between all Borrelia (and B. afzelii alone) and R. helvetica, as well as between N. mikurensis and R. helvetica (Table 3). On the other hand, significant positive associations were found between Borrelia (and particularly B. afzelii) and N. mikurensis and between Borrelia and Babesia spp. (Table 3). These findings lead us to the hypothesis that B. afzelii, N. mikurensis, and Babesia might share the same reservoir hosts, while $R$. helvetica is maintained in other enzootic cycles.

\section{SEASONAL DYNAMICS}

Further evidence for our hypothesis came from the seasonality modeling of the infection prevalence. This indicated a variation in the same phase for B. afzelii, N. mikurensis and Babesia spp. on the one side and for non-afzelii B. burgdorferi and R. helvetica on another (Figure 6). That means that the infection peak in questing ticks is different for different pathogens, further suggesting that they were acquired from the distinct vertebrate hosts. Scientific literature confirms this. Rodents are known to be competent transmission hosts for B. afzelii (Gern and Humair, 2002; Hanincova et al., 2003a) and B. microti (Gray et al., 2002), and 
they have been designated as potential reservoirs for N. mikurensis (Ginsberg, 2008; Andersson and Raberg, 2011). On the other hand, non-afzelii Borrelia, like B. garinii and B. valaisiana have been shown to be associated with birds (Gern and Humair, 2002; Hanincova et al., 2003b), while a study of de la Fuente and coworkers (De La Fuente et al., 2005) found that A. phagocytophilum infections occurred in deer, cattle and various bird species, meaning that birds might serve as reservoirs for both these bacteria. $R$. helvetica was previously found at high rates in both rodents (29\%) and roe deer (19\%) (Sprong et al., 2009). The fact that $R$. helvetica was negatively associated with $B$. afzelii, although they might share the same hosts, is possibly due to that the former is transovarially transmitted in ticks which act thus as both vectors and reservoirs of the rickettsiae (Sprong et al., 2009); therefore, they alone can be responsible for the maintenance of the bacteria, without the intervention of a rodent host in the cycle. Hence, our findings are not coincidental, and indicate that certain coinfections are more likely to occur than the others, given particular combinations of vertebrate hosts.

Although previous meta-analyses indicate that coinfection and co-exposure for some of the TBPs appear to occur somewhat unpredictably across different areas and different hosts (Nieto and Foley, 2009), it is anticipated that future wildlife studies will help define geographical risks of coinfection and provide insight into the dynamics of infection within reservoir hosts.

\section{CONCLUSION}

We have shown that ticks and the five genera of TBPs have a ubiquitous distribution in The Netherlands, with the few absence point presumably determined by the small number of collected ticks. The pathogens were found in sites all over The Netherlands, encompassing a variety of habitats, from open areas such as dune and heather to deciduous or coniferous forests.

This study brings valuable information on the prevalence, geographic distribution and temporal variation of B. burgdorferi s.l., R. helvetica, N. mikurensis, A. phagocytophilum and Babesia spp. in questing $I$. ricinus. Due to their omnipresence, we underline the need to consider all of these pathogens when evaluating the risks of infection and subsequently of disease following a tick bite.

Whereas the incidence of Lyme disease is on the rise, other tick-borne diseases remain heavily unreported, and even

\section{REFERENCES}

Ackermann, R., Kabatzki, J., Boisten, H. P., Steere, A. C., Grodzicki, R. L., Hartung, S., et al. (1984). Ixodes ricinus spirochete and European erythema chronicum migrans disease. Yale J. Biol. Med. 57, 573-580.

Altschul, S. F., Gish, W., Miller, W., Myers, E. W., and Lipman, D. J. (1990). Basic local alignment search tool. J. Mol. Biol. 215, 403-410.

Andersson, M., and Raberg, L. (2011). Wild rodents and novel human pathogen candidatus Neoehrlichia mikurensis, Southern Sweden. Emerg. Infect. Dis. 17, 1716-1718. doi: 10.3201/eid1709.101058
Barbour, A. G., Burgdorfer, W., Hayes, S. F., Péter, O., and Aeschlimann, A. (1983). Isolation of a cultivable spirochete from Ixodes ricinus ticks of Switzerland Curr. Microbiol. 8, 123-126. doi: 10.1007/ BF01566969

Belongia, E. A. (2002). Epidemiology and impact of coinfections acquired from Ixodes ticks. Vector Borne Zoonotic Dis. 2, 265-273. doi: $10.1089 / 153036602321653851$

Bonnet, S., Jouglin, M., L'hostis, M., and Chauvin, A. (2007). Babesia sp. EU1 from roe deer and transmission within Ixodes ricinus. Emerg. Infect. Dis. 13,

knowledge on the human exposure to them is scarce. Our study suggests that there are pathogens positively associated with Borrelia (i.e., N. mikurensis and Babesia spp.) in questing ticks. This strengthens the idea of established enzootic cycles (common reservoir hosts) in which these microorganisms are maintained, and it is consequently possible that they might follow the same upward trend as the Lyme spirochetes. In the case of N. mikurensis we have in fact witnessed the beginning of what might be a following upward trend.

Human activity in any natural habitat is accordingly accompanied by an imminent risk of exposure to any of the pathogens. Although the risk, as measured by the density of infected ticks, may vary in time and space, its driving factor appears to be the tick density/activity. It is therefore possible that the risk of exposure to TBPs would be minimized by developing effective and sustainable methods for the control of Ixodes ricinus populations.

\section{AUTHOR CONTRIBUTIONS}

Hein Sprong, Willem Takken, and Joke van der Giessen designed parts of the study. Catharina B. Maassen and Willem Takken organized the collection of ticks and field data. Manoj Fonville performed lab tests and laboratory analyses. Elena Claudia Coipan and Setareh Jahfari analysed data and performed statistical analysis. Katsuhisa Takumi performed mathematical analyses. Hein Sprong, Joke van der Giessen, and Willem Takken supervised different parts of study. Elena Claudia Coipan and Hein Sprong wrote the final manuscript. All authors read and approved the final manuscript.

\section{ACKNOWLEDGMENTS}

The authors are very grateful to the volunteers and co-workers of the Central Veterinary Institute of Wageningen University and Research Centre, particularly Cor Gaasenbeek and Albert de Boer who have dedicated much time and effort to monthly collections of ticks. We are grateful to Christa Drenth for her excellent technical assistance and to Ellen Tijsse-Klasen and Arno Swart for their support and stimulating discussions. This study was financially supported by the Dutch Food and Consumer Product Safety Authority (NVWA), Wageningen University and Research Centre (WUR), and by the Ministry of Health, Welfare and Sport (VWS).

1208-1210. doi: 10.3201/eid1308. 061560

Burri, C., Dupasquier, C., Bastic, V., and Gern, L. (2011). Pathogens of emerging tick-borne diseases, Anaplasma phagocytophilum, Rickettsia spp., and Babesia spp., in ixodes ticks collected from rodents at four sites in Switzerland (Canton of Bern). Vector Borne Zoonotic Dis. 11, 939-944. doi: 10.1089/vbz.2010.0215

Christova, I., Van De Pol, J., Yazar, S., Velo, E., and Schouls, L. (2003). Identification of Borrelia burgdorferi sensu lato, Anaplasma and Ehrlichia species, and spotted fever group Rickettsiae in ticks from Southeastern Europe. Eur. J. Clin. Microbiol. Infect. Dis. 22, 535-542. doi: 10.1007/s10096-003-0988-1

Coipan, E. C., Fonville, M., TijsseKlasen, E., Van Der Giessen, J. W., Takken, W., Sprong, H., et al. (2013). Geodemographic analysis of Borrelia burgdorferi sensu lato using the 5S-23S rDNA spacer region. Infect. Genet. Evol. 17C, 216-222. doi: 10.1016/j.meegid.2013.04.009

Collares-Pereira, M., Couceiro, S., Franca, I., Kurtenbach, K., Schafer, S. M., Vitorino, L., et al. (2004). First isolation of Borrelia lusitaniae from a human patient. J. Clin. 
Microbiol. 42, 1316-1318. doi: 10.1128/JCM.42.3.1316-1318.2004

De Carvalho, I. L., Fonseca, J. E., Marques, J. G., Ullmann, A., Hojgaard, A., Zeidner, N., et al. (2008). Vasculitislike syndrome associated with Borrelia lusitaniae infection. Clin. Rheumatol. 27, 1587-1591. doi: 10.1007/s10067-008-1012-z

De La Fuente, J., Naranjo, V., RuizFons, F., Hofle, U., Fernandez De Mera, I. G., Villanua, D., et al. (2005). Potential vertebrate reservoir hosts and invertebrate vectors of Anaplasma marginale and A. phagocytophilum in central Spain. Vector Borne Zoonotic Dis. 5, 390-401. doi: 10.1089/vbz.2005.5.390

Dister, S. W., Fish, D., Bros, S. M., Frank, D. H., and Wood, B. L. (1997). Landscape characterization of peridomestic risk for Lyme disease using satellite imagery. Am. $J$. Trop. Med. Hyg. 57, 687-692.

Diza, E., Papa, A., Vezyri, E., Tsounis, S., Milonas, I., and Antoniadis, A. (2004). Borrelia valaisiana in cerebrospinal fluid. Emerg. Infect. Dis. 10, 1692-1693. doi: 10.3201/eid1009.030439

Duh, D., Petrovec, M., and AvsicZupanc, T. (2005). Molecular characterization of human pathogen Babesia EU1 in Ixodes ricinus ticks from Slovenia. J. Parasitol. 91, 463-465. doi: 10.1645/GE-394R

Gassner, F., Van Vliet, A. J., Burgers, S. L., Jacobs, F., Verbaarschot, P., Hovius, E. K., et al. (2011). Geographic and temporal variations in population dynamics of Ixodes ricinus and associated Borrelia infections in The Netherlands. Vector Borne Zoonotic Dis. 11, 523-532. doi: 10.1089/vbz.2010. 0026

Gern, L., and Humair, P. F. (2002). "Ecology of Borrelia burgdorferi sensu lato in Europe," in Lyme Borreliosis: Biology, Epidemiology and Control, eds J. S. Gray, O. Kahl, R. S. Lane, and G. Stanek (Wallingford, Oxon: CAB International), 149-174. doi: 10.1079/9780851996325.0149

Ginsberg, H. S. (2008). Potential effects of mixed infections in ticks on transmission dynamics of pathogens: comparative analysis of published records. Exp. Appl. Acarol. 46, 29-41. doi: 10.1007/s10493-008-9175-5

Glass, G. E., Amerasinghe, F. P., Morgan, J. M. 3rd., and Scott, T. W. (1994). Predicting Ixodes scapularis abundance on white-tailed deer using geographic information systems. Am. J. Trop. Med. Hyg. 51, 538-544.

Glass, G. E., Schwartz, B. S., Morgan, J. M. 3rd., Johnson, D. T., Noy, P. M., and Israel, E. (1995). Environmental risk factors for Lyme disease identified with geographic information systems. Am. J. Public Health 85, 944-948. doi: 10.2105/AJPH.85.7.944

Gray, J., Von Stedingk, L. V., Gurtelschmid, M., and Granstrom, M. (2002). Transmission studies of Babesia microti in Ixodes ricinus ticks and gerbils. J. Clin. Microbiol. 40, 1259-1263. doi: 10.1128/JCM.40.4.1259-1263.2002

Hanincova, K., Schafer, S. M., Etti, S., Sewell, H. S., Taragelova, V., Ziak, D., et al. (2003a). Association of Borrelia afzelii with rodents in Europe. Parasitology 126, 11-20.

Hanincova, K., Taragelova, V., Koci, J., Schafer, S. M., Hails, R. Ullmann, A. J., et al. (2003b). Association of Borrelia garinii and $B$. valaisiana with songbirds in Slovakia. Appl. Environ. Microbiol. 69, 2825-2830. doi: 10.1128/AEM.69.5.2825-2830.2003 Hartelt, K., Oehme, R., Frank, H., Brockmann, S. O., Hassler, D., and Kimmig, P. (2004). Pathogens and symbionts in ticks: prevalence of Anaplasma phagocytophilum (Ehrlichia sp.), Wolbachia sp., Rickettsia sp., and Babesia sp. in Southern Germany. Int. J. Med. Microbiol. 293(Suppl. 37), 86-92. doi: 10.1016/S1433-1128(04)80013-5

Heyman, P., Cochez, C., Hofhuis, A., Van Der Giessen, J., Sprong, H., Porter, S. R., et al. (2010). A clear and present danger: tickborne diseases in Europe. Expert Rev. Anti Infect. Ther. 8, 33-50. doi: 10.1586/eri.09.118

Hildebrandt, A., Pauliks, K., Sachse, S., and Straube, E. (2010). Coexistence of Borrelia spp. and Babesia spp. in Ixodes ricinus ticks in Middle Germany. Vector Borne Zoonotic Dis. 10, 831-837. doi: 10.1089/vbz.2009. 0202

Hofhuis, A., Van Der Giessen, J. W., Borgsteede, F. H., Wielinga, P. R., Notermans, D. W., and Van Pelt, W. (2006). Lyme borreliosis in the Netherlands: strong increase in GP consultations and hospital admissions in past 10 years. Euro Surveill. 11:E060622.

Holden, K., Hodzic, E., Feng, S., Freet, K. J., Lefebvre, R. B., and Barthold, S. W. (2005). Coinfection with Anaplasma phagocytophilum alters Borrelia burgdorferi population distribution in $\mathrm{C} 3 \mathrm{H} / \mathrm{HeN}$ mice. Infect. Immun. 73, 3440-3444. doi 10.1128/IAI.73.6.3440-3444.2005

Hugli, D., Moret, J., Rais, O., Moosmann, Y., Erard, P., Malinverni, R., et al. (2009). Tick bites in a Lyme borreliosis highly endemic area in Switzerland. Int. J. Med. Microbiol. 299, 155-160. doi: 10.1016/j.ijmm.2008. 06.001

Ismail, N., Bloch, K. C., and McBride, J. W. (2010). Human ehrlichiosis and anaplasmosis. Clin. Lab. Med. 30, 261-292. doi: 10.1016/j.cll.2009.10.004

Jahfari, S., Fonville, M., Hengeveld, P., Reusken, C., Scholte, E. J., Takken, W. et al. (2012). Prevalence of Neoehrlichia mikurensis in ticks and rodents from North-west Europe. Parasit. Vectors 5:74. doi 10.1186/1756-3305-5-74

Kitron, U., and Kazmierczak, J. J. (1997). Spatial analysis of the distribution of Lyme disease in Wisconsin. Am. J. Epidemiol. 145, 558-566. doi 10.1093/oxfordjournals.aje.a009145

Kjemtrup, A. M., and Conrad, P. A. (2000). Human babesiosis: an emerging tick-borne disease. Int. J. Parasitol. 30, 1323-1337. doi 10.1016/S0020-7519(00)00137-5

Koci, J., Movila, A., Taragel'Ova, V., Toderas, I., Uspenskaia, I., Derdakova, M., et al. (2007). First report of Anaplasma phagocytophilum and its co-infections with Borrelia burgdorferi sensu lato in Ixodes ricinus ticks (Acari: Ixodidae) from Republic of Moldova. Exp. Appl. Acarol. 41, 147-152. doi: 10.1007/s10493-007-9048-3

Lommano, E., Bertaiola, L., Dupasquier, C., and Gern, L. (2012). Infections and coinfections of questing Ixodes ricinus ticks by emerging zoonotic pathogens in Western Switzerland. Appl. Environ. Microbiol. 78, 4606-4612. doi 10.1128/AEM.07961-11

Lotric-Furlan, S., Petrovec, M., AvsicZupanc, T., Nicholson, W. L., Sumner, J. W., Childs, J. E., et al. (1998). Human ehrlichiosis in central Europe. Wien. Klin. Wochenschr. $110,894-897$

Margos, G., Vollmer, S. A., Cornet, M., Garnier, M., Fingerle, V., Wilske, B., et al. (2009). A new Borrelia species defined by multilocus sequence analysis of housekeeping genes. Appl. Environ. Microbiol. 75, 5410-5416. doi 10.1128/AEM.00116-09

Margos, G., Vollmer, S. A., Ogden, N. H., and Fish, D. (2011).
Population genetics, taxonomy, phylogeny and evolution of Borrelia burgdorferi sensu lato. Infect. Genet. Evol. 11, 1545-1563. doi 10.1016/j.meegid.2011.07.022

Nicholson, M. C., and Mather, T. N. (1996). Methods for evaluating Lyme disease risks using geographic information systems and geospatial analysis. J. Med. Entomol. 33, 711-720.

Nieto, N. C., and Foley, J. E. (2009). Meta-analysis of coinfection and coexposure with Borrelia burgdorferi and Anaplasma phagocytophilum in humans, domestic animals, wildlife, and Ixodes ricinus-complex ticks. Vector Borne Zoonotic Dis. 9, 93-102. doi: 10.1089/vbz.2008.0072

Parola, P., and Raoult, D. (2001). Ticks and tickborne bacterial diseases in humans: an emerging infectious threat. Clin. Infect. Dis. 32, 897-928. doi: 10.1086/319347

Randolph, S. E. (2001). The shifting landscape of tick-borne zoonoses: tick-borne encephalitis and Lyme borreliosis in Europe. Philos. Trans. R. Soc. Lond. B Biol. Sci. 356, 1045-1056. doi: 10.1098/rstb.2001.0893

Rauter, C., and Hartung, T. (2005). Prevalence of Borrelia burgdorferi sensu lato genospecies in Ixodes ricinus ticks in Europe: a metaanalysis. Appl. Environ. Microbiol. 71, 7203-7216. doi: $\quad$ 10.1128/AEM.71.11.72037216.2005

Reye, A. L., Hubschen, J. M., Sausy, A., and Muller, C. P. (2010). Prevalence and seasonality of tickborne pathogens in questing Ixodes ricinus ticks from Luxembourg. Appl. Environ. Microbiol. 76 2923-2931. doi: 10.1128/AEM. 03061-09

Rydkina, E., Roux, V., Rudakov, N., Gafarova, M., Tarasevich, I., and Raoult, D. (1999). New Rickettsiae in ticks collected in territories of the former soviet union. Emerg. Infect. Dis. 5, 811-814. doi: 10.3201/eid0506. 990612

Schouls, L. M., Van De Pol, I., Rijpkema, S. G., and Schot, C. S. (1999). Detection and identification of Ehrlichia, Borrelia burgdor feri sensu lato, and Bartonella species in Dutch Ixodes ricinus ticks. J. Clin. Microbiol. 37, 2215-2222.

Severinsson, K., Jaenson, T. G. Pettersson, J., Falk, K., and Nilsson, K. (2010). Detection and prevalence of Anaplasma phagocytophilum and Rickettsia helvetica in Ixodes 
ricinus ticks in seven study areas in Sweden. Parasit. Vectors 3:66. doi: 10.1186/1756-3305-3-66

Smith, R., and Takkinen, J. (2006). Lyme borreliosis: europe-wide coordinated surveillance and action needed? Euro Surveill. 11:E060622.

Sprong, H., Wielinga, P. R., Fonville, M., Reusken, C., Brandenburg, A. H., Borgsteede, F., et al. (2009). Ixodes ricinus ticks are reservoir hosts for Rickettsia helvetica and potentially carry flea-borne Rickettsia species. Parasit. Vectors 2:41. doi: 10.1186/1756-3305-2-41

Stanek, G., Wormser, G. P., Gray, J., and Strle, F. (2012). Lyme borreliosis. Lancet 379, 461-473. doi: 10.1016/S0140-6736(11)60103-7

Svendsen, C. B. (2011). Is sarcoidosis a rickettsiosis? Dan. Med. Bull. 58:B4249.

Swanson, S. J., Neitzel, D., Reed, K. D., and Belongia, E. A. (2006). Coinfections acquired from ixodes ticks. Clin. Microbiol. Rev. 19, 708-727. doi: 10.1128/CMR.00011-06

Thomas, V., Anguita, J., Barthold, S. W., and Fikrig, E. (2001). Coinfection with Borrelia burgdorferi and the agent of human granulocytic ehrlichiosis alters murine immune responses, pathogen burden, and severity of Lyme arthritis. Infect. Immun. 69, 3359-3371. doi: 10.1128/IAI.69.5. 3359-3371.2001

Tijsse-Klasen, E., Fonville, M., Gassner, F., Nijhof, A. M., Hovius, E. K., Jongejan, F., et al. (2011a). Absence of zoonotic Bartonella species in questing ticks: first detection of Bartonella clarridgeiae and Rickettsia felis in cat fleas in the Netherlands. Parasit. Vectors 4:61. doi: 10.1186/1756-3305-4-61

Tijsse-Klasen, E., Fonville, M., Reimerink, J. H., Spitzen-Van Der Sluijs, A., and Sprong, H. (2010). Role of sand lizards in the ecology of Lyme and other tick-borne diseases in the Netherlands. Parasit. Vectors 3:42. doi: 10.1186/17563305-3-42

Tijsse-Klasen, E., Jacobs, J. J., Swart, A., Fonville, M., Reimerink, J. H., Brandenburg, A. H., et al. (2011b). Small risk of developing symptomatic tick-borne diseases following a tick bite in the Netherlands. Parasit. Vectors 4:17. doi: 10.1186/1756-3305-4-17

Van Dobbenburgh, A., Van Dam, A. P., and Fikrig, E. (1999). Human granulocytic ehrlichiosis in western Europe. N. Engl. J. Med. 340, 1214-1216. doi: 10.1056/NEJM199904153401517

Vannier, E., and Krause, P. J. (2012). Human Babesiosis. N. Engl. J. Med. 366, 2397-2407. doi: 10.1056/NEJMra1202018

Wang, G., Van Dam, A. P., and Dankert, J. (1999). Phenotypic and genetic characterization of a novel Borrelia burgdorferi sensu lato isolate from a patient with lyme borreliosis. J. Clin. Microbiol. 37, 3025-3028.

Wielinga, P. R., Fonville, M., Sprong, H., Gaasenbeek, C., Borgsteede, F., and Van Der Giessen, J. W. (2009). Persistent detection of Babesia EU1 and Babesia microti in Ixodes ricinus in the Netherlands during a 5-year surveillance: 2003-2007. Vector Borne Zoonotic Dis. 9, 119-122. doi: 10.1089/vbz.2008. 0047

Wielinga, P. R., Gaasenbeek, C., Fonville, M., De Boer, A., De Vries, A., Dimmers, W., et al. (2006). Longitudinal analysis of tick densities and Borrelia, Anaplasma, and Ehrlichia infections of Ixodes ricinus ticks in different habitat areas in The Netherlands. Appl. Environ. Microbiol. 72,
7594-7601. doi: 10.1128/AEM. 01851-06

Conflict of Interest Statement: The authors declare that the research was conducted in the absence of any commercial or financial relationships that could be construed as a potential conflict of interest.

Received: 28 April 2013; accepted: 09 July 2013; published online: 30 July 2013. Citation: Coipan EC, Jahfari S, Fonville $M$, Maassen $C B$, van der Giessen J, Takken W, Takumi $K$ and Sprong $H$ (2013) Spatiotemporal dynamics of emerging pathogens in questing Ixodes ricinus. Front. Cell. Infect. Microbiol. 3:36. doi: 10.3389/fcimb.2013.00036 Copyright (c) 2013 Coipan, Jahfari, Fonville, Maassen, van der Giessen, Takken, Takumi and Sprong. This is an open-access article distributed under the terms of the Creative Commons Attribution License (CC BY). The use, distribution or reproduction in other forums is permitted, provided the original author(s) or licensor are credited and that the original publication in this journal is cited, in accordance with accepted academic practice. No use, distribution or reproduction is permitted which does not comply with these terms. 